\title{
Wireless Sensor Network based on OCDMA for closed environments
}

\author{
E. Poves*, J. A. Martin-Gonzalez ${ }^{\dagger}$, G. del Campo-Jimenez* and F. J. Lopez-Hernandez* \\ ${ }^{*}$ Centro de Domótica Integral - CeDInt \\ Universidad Politécnica de Madrid \\ 28223 Pozuelo de Alarcón (Madrid, Spain) \\ †Dept. Matemáticas y Computación \\ Universidad de Burgos \\ 09001 Burgos (Spain)
}

\begin{abstract}
An infrared optical wireless system is presented, consisting on autonomous remote nodes communicating with a central node. The network is designed for telecommand/telemetry purposes, comprising a large number of nodes at a low data rate . Simultaneous access is granted by using CDMA techniques, and an appropriate selection of the code family can also keep power consumption to a minimum.
\end{abstract}

Index Terms-Code Division Multiple-Access (CDMA), Optical CDMA (OCDMA), Direct-Sequence Spread-Spectrum (DS/SS), wireless communications, wireless sensor networks (WSN), Random Optical Codes (ROCs), telecommand, telemetry.

\section{INTRODUCTION}

In the late years, optical wireless has been considered as an alternative to wired and radiofrequency communications. In environments with special restrictions on electromagentic interference (EMI), such as aircrafts or hospitals, this technique provides mobility without affecting other systems' performance. Previous studies [1], [2] have compared the performance of radiofrequency and infrared optical communications, and channel estimations [3] demonstrate that the effect of the channel can be neglected in small environments if chip rates are lower than some hundreds of $\mathrm{MHz}$.

Infrared (IR) systems are commonly used for point-to-point, line-of-sight links. But using Spread-Spectrum techniques, such as OCDMA, independent and simultaneous channel access can be granted for several network nodes [4]. Different code families have beeen proposed [5], [6], [7], each with particular properties that affect system's parameters like the bit-error rate (BER) or the maximum data rate achievable.

In this work a telecommand/telemetry (TC/TM) network consisting on 25 autonomous nodes is described. All nodes are capable of communicating with a central node through a full-duplex asynchronous optical wireless channel.

\section{SySTEM OVERVIEW}

The objective of this development is to communicate several nodes in a small and closed volume, assuming traveling distances up to 2-3 meters. The line-of-sight (LoS) is not guaranteed and working in diffusion is required, as the position of the nodes should be constraint-free. Power needs will also be kept to a minimum, enabling battery operation and long lifetimes. Data rate is $1 \mathrm{kbps}$, which is well suited for the network purposes.

The large number of nodes in the system (25 units were tested simultaneously) makes OCDMA a good choice to simplify the system design and operation. This technique controls multiple user interference (MUI), making medium access control protocols not necessary to ensure the desired behavior. Furthermore, the system is given a great flexibility to accommodate new nodes without affecting network characteristics such as the bandwidth available. For the intended application, spread-spectrum techniques offer an additional security as all data goes through an encription when transmitted.

Terminal nodes have been implemented using a modular structure to allow an easy reconfiguration to test different target applications. A logic module consisting on a low-power CPLD controls the communication process, data acquisition and the node operation. This module is easily replaceable to allow upgrades if new functionalities are required and a different logic device is to be used. A separate communication module performs the signal conversion from optical to electrical and viceversa, ensuring sent and received signal's integrity. The data acquisition module is beyond this demonstrator's objectives, and all nodes are prepared to simulate both sensor and actuator features by using an user-selectable switch and a RGB LED.

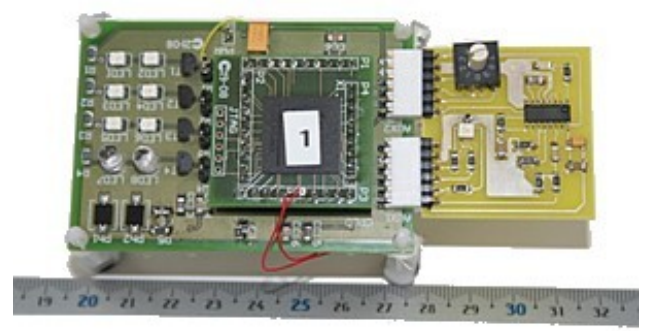

Fig. 1. Node implementation

Central node stores and analyzes all gathered data and controls the communications, requiring bigger processing capabilities than the individual nodes designed. It is based on a commercial control and acquisition system and integrates a microprocessor running a real-time OS with an FPGA 
backplane to interface with external signals. To simplify the node's operation, channel synchronization is implemented on the central node which stores a delay reference for each terminal node. This delay is updated upon data reception and will be used for transmission to ensure the node can properly recover the data.

Random Optical Codes are used for data coding. As presented in [7], this family has better performance than prime codes for large number of users. A total of 50 codewords are needed to allow full-duplex simultaneous access, 25 for the uplinks and 25 for the downlinks. To obtain a target error probability of $10^{-7}$, codes of length $L=900$ and weight $w=20$ are required [8]. This means each bit will be divided into 900 slots (named chips) and 20 of them will be "marked". Given that the data rate is $1 \mathrm{kbps}$, the system will be operating at $900 \mathrm{kHz}$ which is well below the limit imposed by the channel response [3] and multipath dispersion effect on the communications can be neglected.

\section{DEMONSTRATOR}

The whole system can be randomly deployed on a table as shown in Fig.2. Central node's emitter and receiver are placed at a variable distance over the table to ensure visibility from all the nodes (which is not necessary in closed environments). Nodes are powered by 2 standard AA batteries $(3 \mathrm{~V})$, except for the central node which uses its own power supply unit.

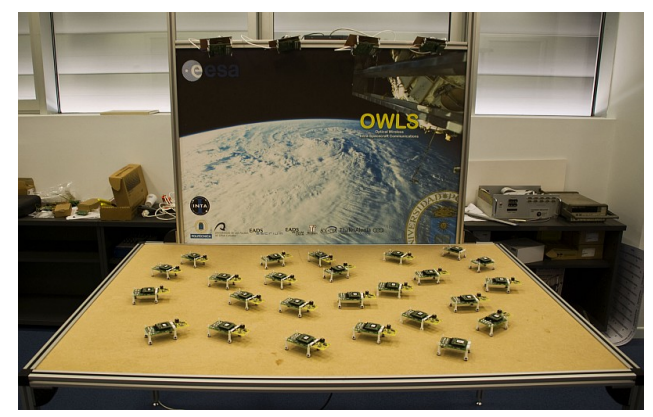

Fig. 2. System overview

Central node provides a system monitor application which allows the operator to inspect the network status and set the data to be sent to each node. All 25 nodes are presented with the actual values coming from the user-selectable switch, and different colors can be selected on the monitor application that will be sent to the node and shown on the attached RGB LED. This way a simple visual inspection is possible over the whole system operation.

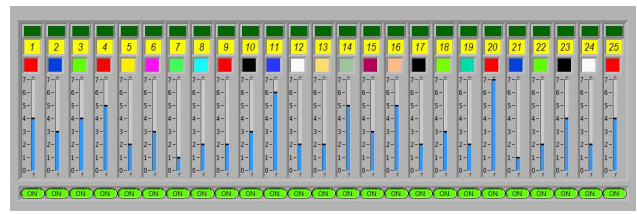

Fig. 3. System monitor application

\section{CONCLUSIONS}

A test system has been designed and implemented to validate the behavior of OCDMA techniques in closed environments. A broad area is covered and nodes can be deployed in random positions thanks to the diffusive design. Random Optical Codes (ROC) have been tested for environments with a large number of simultaneous users, validating the results from previous simulations and assessing its good properties for these applications. Also, the possibility of dinamically assigning codewords depending on the workload shall be studied to maximize the throughput and optimize the bandwith utilization.

\section{ACKNOWLEDGMENTS}

This work has been developed as part of the project Optical Wireless for Intra-Spacecraft Communications (ESA Contract 19545) in collaboration with Instituto Nacional de Tecnología Aeroespacial (INTA), and spanish Ministry of Education's national plan (TEC2006-13887-C05-01).

\section{REFERENCES}

[1] D. J. T. Heatley and I. Neild, "Optical wireless - the promise and the reality," IEE Colloquium on Optical Wireless Communications, June 1999.

[2] A. M. Street, P. N. Stavrinou, D. C. O'brien, and D. J. Edwards, "Indoor optical wireless systems - a review," Optical and Quantum Electronics, vol. 29 , no. 3, pp. 349-378, March 1997.

[3] J. A. Rabadán, M. A. Bacallado, F. Delgado, S. T. Pérez, and R. PérezJiménez, "Experimental characterization of a direct-sequence spreadspectrum optical wireless system based on pulse-conformation techniques for in-house communications," IEEE Transactions on Consumer Electronics, vol. 50, no. 2, May 2004.

[4] R. D. J. V. Nee, "Spread-spectrum code and carrier synchronization errors caused by multipath and interference," IEEE Transactions on Aerospace and Electronic Systems, vol. 29, no. 4, October 1993.

[5] J. A. Salehi, "Code division multiple-access techniques in fiber optics networks-part I: Fundamental principles," IEEE Transactions on Communications, vol. 37, no. 8, August 1989.

[6] G. C. Yang and W. C. Kwong, "Performance analisis of optical cdma with prime codes," Electronic Letters, vol. 31, no. 7, pp. 569-570, March 1995.

[7] J. A. Martin-Gonzalez, E. Poves, and F. J. Lopez-Hernandez, "Random optical codes used in optical networks," IET Communications, vol. 3, no. 8, pp. 1392-1401, August 2009.

[8] E. Poves, J. A. Martin-Gonzalez, and F. J. Lopez-Hernandez, "Use of optical orthogonal codes for intra-spacecraft communications," in International Conference on Transparent Optical Networks - Mediterranean Winter ICTON-MW'07, Sousse (Túnez), Dec. 2007.

[9] J. A. Salehi and C. A. Brackett, "Code division multiple-access techniques in fiber optics networks-part II: Systems performance analysis," IEEE Transactions on Communications, vol. 37, August 1989.

[10] J. A. Martin-Gonzalez, E. Poves, and F. J. Lopez-Hernandez, "Random optical codes in an intra-satellite optical wireless network," in International Conference on Transparent Optical Networks - Mediterranean Winter ICTON-MW'07, Sousse (Túnez), Dec. 2007.

[11] _ " "Random optical codes for optical code-division multiple-access," in International Conference on Wireless and Optical Communications WOC 2008, Quebec (Canadá), Mayo 2008.

[12] O. Gonzalez, S. Rodriguez, R. Perez-Jimenez, B. R. Mendoza, and A. Ayala, "Error analysis of the simulated impulse response on indoor wireless optical channels using a monte carlo-based ray-tracing algorithm," IEEE Trans. Commun., vol. 52, no. 1, pp. 124-130, Jan. 2005.

[13] J. A. Martin-Gonzalez, E. Poves, and F. J. Lopez-Hernandez, "Probability of error analisis of random optical codes for optical code-division multiple-access," IET Communications, 2009. 